Supporting information

\title{
Fast and broadband photoresponse of few-layer GeSe field-effect transistor with direct bandgaps
}

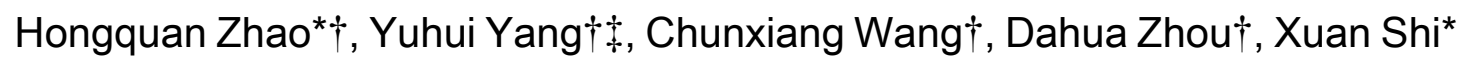

$\dagger$, Yuzhi Li†, Yuliang Mao $\S$

$\dagger$ Chongqing Institute of Green and Intelligent Technology, Chinese Academy of Sciences

$\$$ University of Chinese Academy of Sciences, Beijing 100064, China

$\S$ Hunan Key Laboratory for Micro-Nano Energy Materials and Devices, School of Physics and Optoelectronic Engineering, Xiangtan University, Hunan 411105, People's Republic of China

\section{E-mail: hqzhao@cigit.ac.cn, shixuan@cigit.ac.cn}

KEYWORDS: few-layer GeSe, field effect transistors, photoresponse time, broadband photoresponse, direct bandgaps, ambipolar behavior

\section{Content}

S1 Experimental setup

S2 Molar ratio analysis for laser thinned few-layer GeSe

S3 Laser thinned GeSe device with direct bandgaps

S4 Large photocurrent with high laser power densities

S5 Stability of photocurrent analysis of the FET device

References 


\section{S1 Experimental setup}

A home-made laser scanning confocal microscope equipped with an objective lens of 0.6 numerical aperture and a 532nm cw laser (Cobolt-Samba-100) was used to perform the focused laser scanning thinning process in high vacuum. ${ }^{1}$ Subsequently, fluorescence spectrum measurements were taken in high vacuum on the same stage. Samples were fixed on a cold finger inside a cryostat (ARS-4HW), and the cold finger is fixed on a piezoelectric ceramics (PZT) with $10 \mathrm{~nm}$ of resolution and $5 \mathrm{~mm}$ of marches in the $\mathrm{X}, \mathrm{Y}$, and $\mathrm{Z}$ directions. Figure $\mathrm{S} 1$ show schematically the experimental setup for controlled laser thinning of GeSe nanosheet to few layers and the fluorescence spectrum measurements.

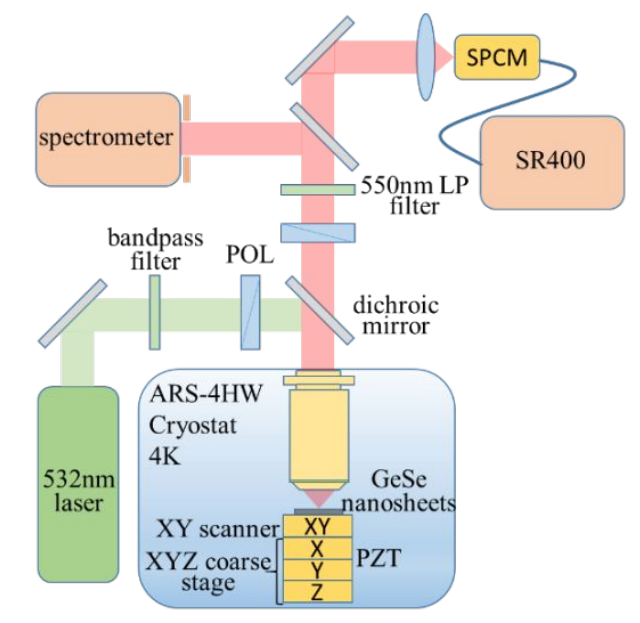

Figure S1: Experimental setup for controlled laser thinning and fluorescence measurements. POL: polarizer, LP: long-pass filter, SPCM: single photon counting module with the mode of count-50c, SR400: a single photon counter made by Stanford Instruments. PZT: piezoelectric ceramics.

\section{S2 Molar ratio analysis for laser thinned few-layer GeSe}

Scanning electron microscope (SEM, JEOL ultra-high resolution JSM-7800F) combined with energy dispersive spectrum (EDS) was performed simultaneously in the pristine region (R1) and the thinned region (R2) in Figure S2 to depict the molar ratio of Ge and Se atoms. Figure S2a shows a molar ratio of $3.53 \%$ and $3.22 \%$ for Ge and Se atoms in R1 region and $0.05 \%$ 
in $\mathrm{R} 2$ region for both $\mathrm{Ge}$ and $\mathrm{Se}$ atoms, respectively, which is well consistent with the stoichiometric ratio of the GeSe as $\sim 1: 1$. Figure $\mathrm{S} 2 \mathrm{~b}$ shows a similar result as Figure $\mathrm{S} 2 \mathrm{a}$, and the Ge and Se molar ratio of 1:1 is achieved after the laser thinning in R2 region. Figure S2c and S2d show the molar ratio of Ge and Se to be 1.14 and 1.28 in the laser thinned region, respectively, indicating very small of Se vacancy is produced in these laser thinning process. This evidence unambiguously illustrates the pure phase of the thinned bottom layer, and a high chemical stability during the thinning process. It also indicate that the Se vacancy is not a fatal problem of the laser thinned GeSe layer that used for photoelectronic devices.

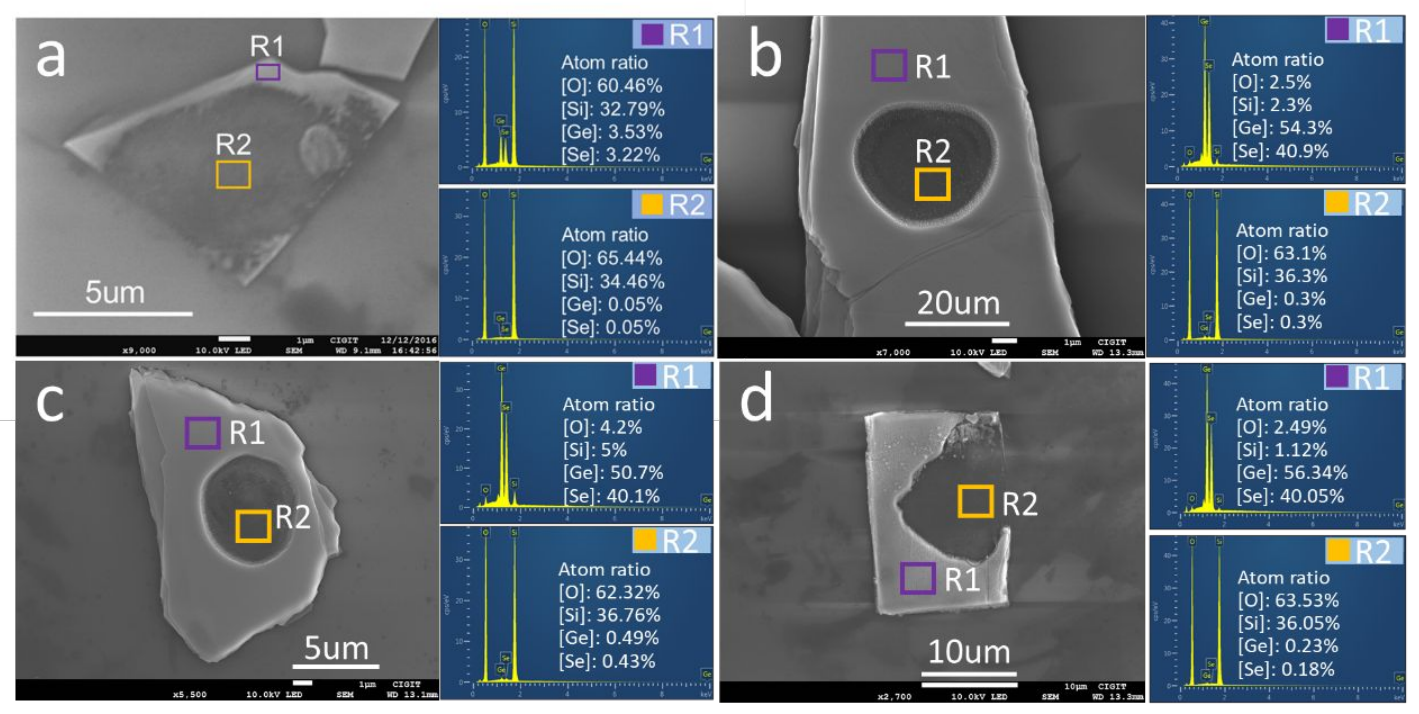

Figure S2: SEM image of four samples and their EDS spectrum measured in the selected pristine regions (R1) and laser thinned regions (R2) in each sample. (a) Element ratios of Ge and Se decreased from $3.53 \%$ and $3.22 \%$ in $\mathrm{R} 1$ region to $0.05 \%$ both for Ge and Se in $\mathrm{R} 2$ region, respectively. (b) Element ratios of Ge and Se decreased from $54.3 \%$ and $40.9 \%$ in R1 region to $0.3 \%$ both for $\mathrm{Ge}$ and $\mathrm{Se}$ in $\mathrm{R} 2$ region, respectively. (c) Element ratios of Ge and $\mathrm{Se}$ decreased from $50.7 \%$ and $40.1 \%$ in R1 region to $0.49 \%$ and $0.43 \%$ for Ge and Se in R2 region, respectively. (d) Element ratios of Ge and Se decreased from $56.43 \%$ and $40.05 \%$ in R1 region to $0.23 \%$ and $0.18 \%$ for Ge and Se in $\mathrm{R} 2$ region, respectively.

\section{S3 Laser thinned GeSe device with direct bandgaps}

Figure S3 (a) show the optical image of a fabricated GeSe two-terminal device, and (b) shows the PL mapping image of the GeSe device after focused laser-scan thinning. The PL mapping 
image of the thinned GeSe device is measured at an excitation laser power density of $0.3 \times 10^{4}$ $\mathrm{W} / \mathrm{cm}^{2}$ with stepping resolution of $0.5 \mathrm{um}$. The laser thined region of the device shows an almost uniform fluorescence intensity. It has been reported that the critical layer number from indirect to direct bandgap transition is happened at the $6^{\text {th }}$ layers, indicating direct bandgap of the present thinned GeSe flake with high homogeneity. ${ }^{2}$
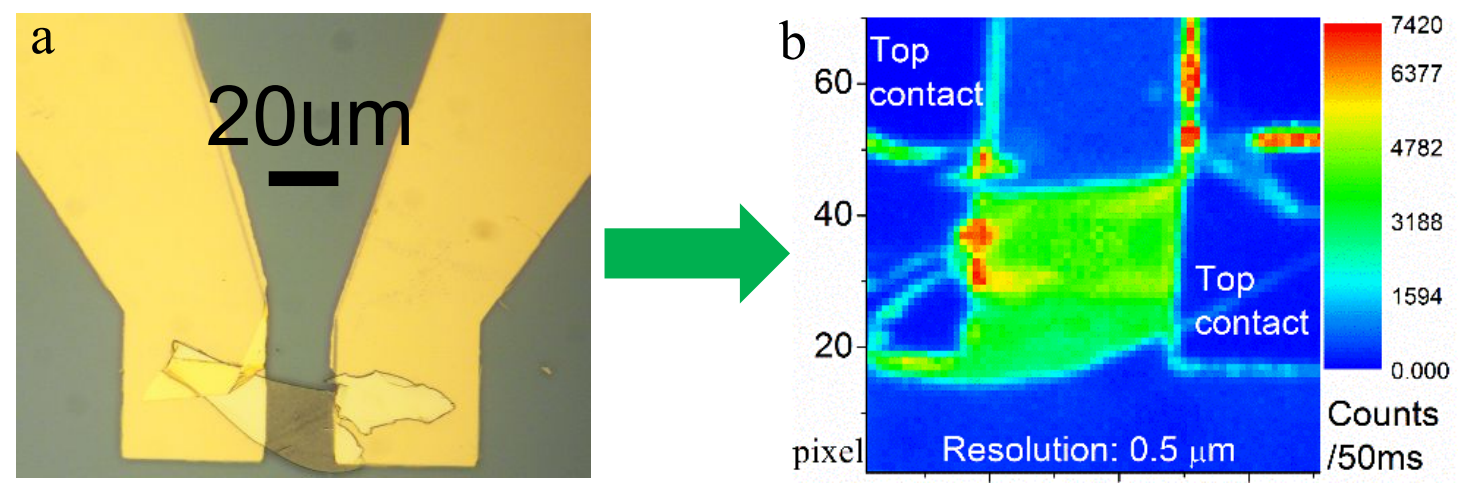

Figure S3: (a) Optical image of a fabricated GeSe two-terminal device, and (b) PL mapping image of the GeSe device after focused laser-scan thinning.

\section{S4 Large photocurrent with high laser power densities}

We measured photocurrents under a $405 \mathrm{~nm}$ laser illumination at a varied effective laser power from $70 \mu \mathrm{W}$ to $700 \mu \mathrm{W}$, as we shown in Figure S4. The focused laser spot size is about

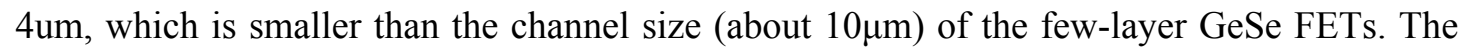
photocurrent approaches $\sim 1 \mu \mathrm{A}$ is measured at $700 \mu \mathrm{W}$ of effective laser power. It is to be noted that we chose to limit the spot size of our laser beam to minimize the interaction with the contacts in an effort to evaluate the intrinsic photo-responsivity of the few-layer GeSe. 

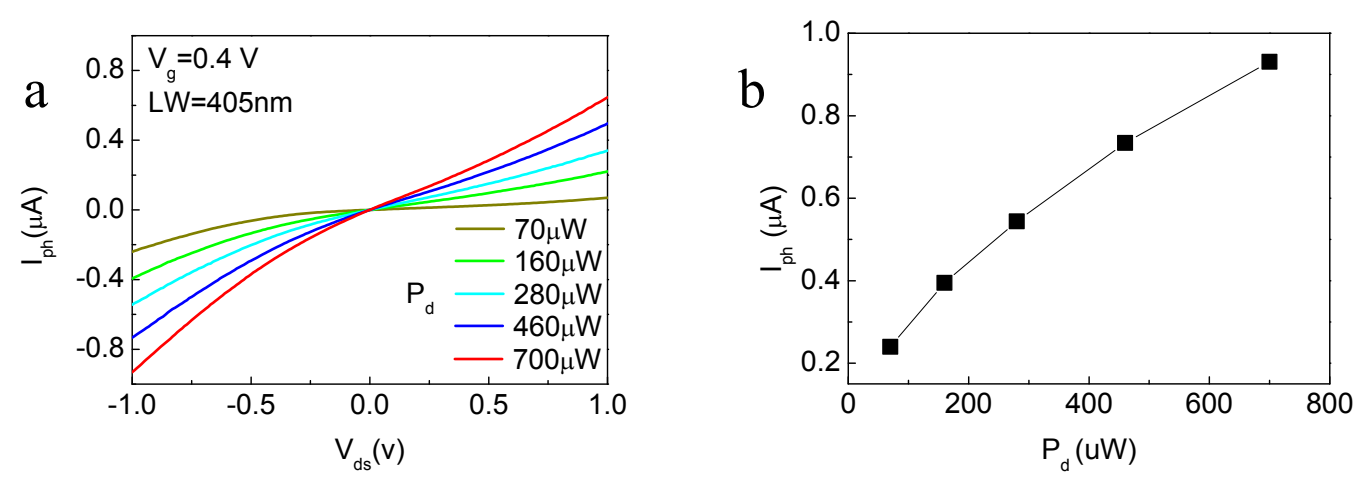

Figure S4: (a) Measured photocurrent at different effective laser power under the illumination of a $405 \mathrm{~nm}$ laser at a gate voltage value of $0.4 \mathrm{~V}$. (b) The photocurrent varied with the effective laser power at the drain-source voltage of $-1 \mathrm{~V}$.

\section{S5 Stability of photocurrent analysis of the FET device}

Stability of the photocurrent response was simply estimated by an extended duration photocurrent measurement by switching the laser on and off. ${ }^{3}$ The duration at the on state was sustained during 50 seconds, Figure S4 shows the photocurrent stability over 600 seconds of continuous operation. Photocurrent intensity oscillations in the direct current (DC) level are obvious. Even though, it is still possible to notice that the photocurrent response is very stable since there is no significant decay on the signal amplitude in each photocurrent duration. The slow variations of the DC level can be produced by thermal drift produced by the heat accumulation on the device, since it is assembled on a thermally insulating substrate..$^{4-5}$ 


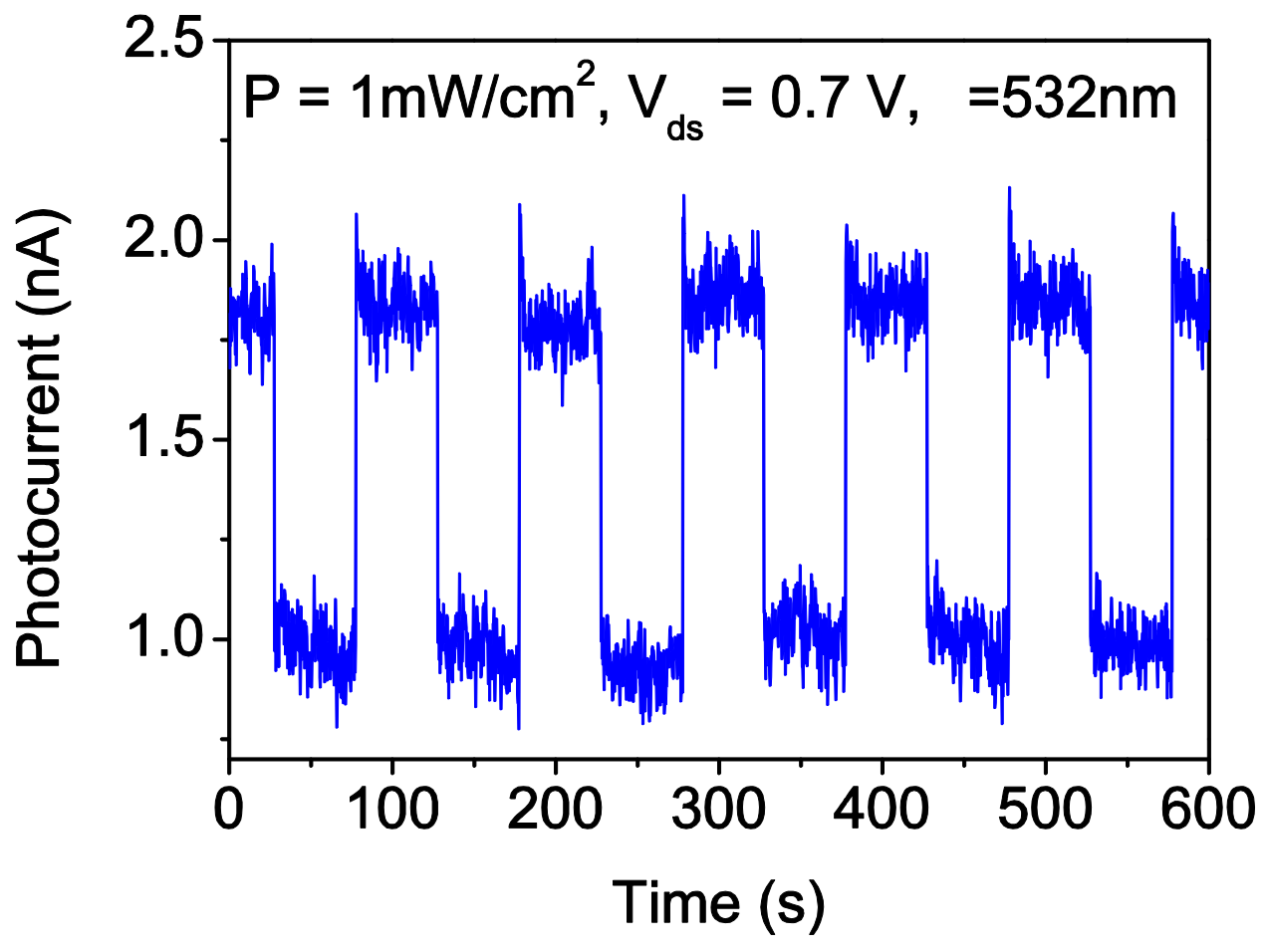

Figure S5: Photocurrent vs. time with alternated turn-on and turn-off laser beam pulse. Good stability of the photocurrent response after almost 600 seconds of operation with $50 \mathrm{~s}$ duty cycles. The laser beam wavelength corresponded to $650 \mathrm{~nm}$ at $1 \mathrm{~mW} / \mathrm{cm}^{2}$ and the device was polarized with $0.7 \mathrm{~V}$. Notice the laser status shifts between on and off, the current increases sharply.

\section{References:}

1. Zhao, H.-Q.; Mao, Y. L.; Mao, X.; Shi, X.; Xu, C. Sh.; Wang, Ch. X.; Zhang, Sh. M.; Zhou,

D. H. Band Structure and Photoelectric Characterization of GeSe Monolayers, Adv. Funct. Mater. 2018, 1704855.

2. Song, X. F.; Zhou, W. H.; Liu, X. H.; Gu, Y.; Zhang, Sh. L. Layer-controlled band alignment, work function and optical properties of few-layer GeSe, Physica B 2017, 519, 90.

3. Perea-López, N.; Elías, A. L.; Berkdemir, A.; Castro-Beltran, A. H.; Gutiérrez, R.; Feng, S.; 
Lv, R.; Hayashi, T.; López-Urías, F.; Ghosh, S.; Muchharla, B.; Talapatra, S.; Terrones, H.; Terrones, M. Photosensor Device Based on Few-Layered WS2 Films, Adv. Funct. Mater. 2013, $23,5511$.

4. Song, J. C. W.; Rudner, M. S.; Marcus, C. M.; Levitov, L. S. Hot Carrier Transport and Photocurrent Response in Graphene, Nano Lett. 2011, 11, 4688.

5. Kung, Sh.-Ch.; van der Veer, W. E.; Yang, F.; Donavan, K. C.; Penner, R. M. $20 \mu \mathrm{s}$ Photocurrent Response from Lithographically Patterned Nanocrystalline Cadmium Selenide Nanowires, Nano Lett. 2010, 10, 1481. 